\title{
Prognostic value of estrogen receptor and progesterone receptor tumor expression in Danish ovarian cancer patients: From the 'MALOVA' Ovarian Cancer Study
}

\author{
ESTRID V.S. HØGDALL ${ }^{1}$, LISE CHRISTENSEN ${ }^{2}$, CLAUS K. HØGDALL ${ }^{3}$, JAN BLAAKAER ${ }^{4}$, \\ SIMON GAYTHER $^{5}$, IAN J. JACOBS ${ }^{5}$, IB JARLE CHRISTENSEN ${ }^{6}$ and SUSANNE K. KJAER ${ }^{1}$
}

\author{
${ }^{1}$ Department of Virus, Hormones and Cancer, Institute of Cancer Epidemiology, Danish Cancer Society, \\ Copenhagen; ${ }^{2}$ Department of Pathology, Bispebjerg Hospital, ${ }^{3}$ The Gynaecologic Clinic, The Juliane Marie Centre, \\ Rigshospitalet, University of Copenhagen, Copenhagen; ${ }^{4}$ Department of Gynecology and Obstetrics, Aarhus University \\ Hospital, Skejby, Aarhus, Denmark; ${ }^{5}$ Institute of Women's Health, University College London, UK; ${ }^{6}$ Department \\ of Surgical Gastroenterology, Hvidovre Hospital, University of Copenhagen, Copenhagen, Denmark
}

Received May 14, 2007; Accepted July 10, 2007

\begin{abstract}
Estrogen and progesterone are important hormones secreted by the ovary acting through specific receptors. Tumor tissue expression profiles of these have demonstrated prognostic value in malignancies such as breast, uterine and prostate cancer. In this study, including tissue samples from 773 Danish patients with an ovarian tumor, we evaluated whether estrogen receptor (ER) and progesterone receptor (PR) expression correlated with clinico-pathological parameters, and a possible prognostic impact on ovarian cancer (OC) patients was investigated. Using tissue array and immunohistochemistry, we analyzed the ER and PR expression levels in tissues from 582 women with OC and 191 women with low malignancy potential (LMP) ovarian tumors. Our results demonstrated that ER was expressed in 30 of the 191 LMP tumors (16\%) and in 207 of the 582 OC (36\%). PR was expressed in 38 LMP tumors (20\%) and in 115 OC (20\%). For both tumor types an excess of positive tumors was found in the serous compared to the mucinous subtype $(\mathrm{p} \leq 0.00001)$. The frequency of ER expressionpositive OC increased with increasing FIGO stage
\end{abstract}

Correspondence to: Dr Estrid Høgdall, Department of Virus, Hormones and Cancer, Institute of Cancer Epidemiology, Danish Cancer Society, Strandboulevarden 49, DK-2100 Copenhagen, Denmark

E-mail: hogdall@dadlnet.dk or estrid@cancer.dk

Abbreviations: OC, ovarian cancer; LMP, low malignant potential; $\mathrm{TA}$, tissue array; ER, estrogen receptor; PR, progesterone receptor; $\mathrm{H} \& \mathrm{E}$, hematoxylin and eosin; CV, coefficient of variation; CI, confidence interval; HR, hazard ratio; MALOVA, Malignant Ovarian Cancer Study; FIGO, The International Federation of Gynaecology and Obstetrics

Key words: tissue array, ovarian cancer, estrogen, progesterone, immunohistochemistry, prognosis $(\mathrm{p}=0.0003)$, and the frequency of PR-positive tumors increased with increasing histological grade $(\mathrm{p}=0.0006)$. In a Cox survival analysis, a tissue ER and PR expression $10 \%$ or higher was found to imply an independent significant advantageous course of patient disease-specific survival (ER: hazard ratio (HR), 0.80 ; 95\% confidence interval (CI), 0.630.99; PR: HR, 0.69; 95\% CI, 0.51-0.94) together with FIGO stage, residual tumor after primary surgery, age at diagnosis and other histological types vs. serous adenocarcinoma. The histological grade of tumor was found to have no independent prognostic value. The prognostic value of ER and PR was found additive with a $\mathrm{HR}$ for patients with high $\mathrm{ER}$ and $\mathrm{PR}$ expression of 0.48 (95\% CI, 0.31-0.74) compared to patients with $<10 \%$ expression for both receptors. In conclusion, our results predict that an elevated expression of ER and PR, alone and in combination, point to a favorable outcome for patients with OC.

\section{Introduction}

The incidence of ovarian cancer (OC) varies widely among different geographic regions and ethnic groups, the highest occurring in Scandinavia, Western Europe and North America and the lowest in Asian countries. Every year more than 600 new cases appear in Denmark, yielding an age-standardized incidence rate of 13.3 per 100,000 women-year (1998-2002), which is one of the highest incidence rates in the world (1).

More than $70 \%$ of the women are diagnosed with latestage disease [International Federation of Gynecology and Obstetrics (FIGO) stage III or stage IV]. For these women the 5 -year overall survival is $<20 \%$, in contrast to women with early stage disease, who hold a corresponding survival of $\sim 90 \%(2,3)$.

Identifying the prognostic factors for $\mathrm{OC}$ is a challenging task. The carcinogenic mechanisms are still unknown. The tumor is clinico-pathologically complex, and studies of large cohorts of cases with complete clinical and pathological data and follow-up are scarce. 
Estrogen and progesterone are important hormones secreted by the ovary acting through specific receptors. A putative direct action of gonadal steroids on ovarian carcinogenesis has been suggested, supported by findings of mRNA transcripts and translated proteins of estrogen receptors (ER) and progesterone receptors (PR) in both normal ovarian tissue and malignant ovarian tumors $(4,5)$. The action of ER is believed to be mediated by the two ER receptors, ER- $\alpha$ and ER- $\beta$, which through differential regulation of gene transcription may exert opposite actions on OC growth and survival (6-8). It is thus conceivable that the expression profiles of ER and PR are related to ovarian tumor behavior or prognosis $(5,9)$, as it has been shown for other tumors such as breast, endometrial and prostate cancer (4,6,9-11).

In contrast to breast cancer, limited and conflicting data concerning ER and PR expression are available for OC. Like ours, these studies have aimed at correlating ER and PR expression with grade and stage of OC and with patient survival $(5,9)$, but the conflicting findings may appear as a result of various factors, e.g. differences in the method to detect receptors, lack of standardization of the scoring system, and status and size of the samples analysed. Although steroid hormones and receptors may have important influence on OC tumorigenesis and progression of $\mathrm{OC}$, comprehensive immunohistochemical analyses of tumoral ER and PR expression in large representative patient collectives are scarce.

Recent progress in methodologies has improved the ability to study somatic alterations in a larger number of tumors, also from archival material, than has previously been possible. The tissue array (TA) technologies are designed to increase the throughput of immunohistochemical analyses of protein expression. This is in contrast to traditional immunohistochemical studies in which larger sections of the tumor are analysed individually $(12,13)$.

This study was aimed at determining the expression of ER and PR in tissue samples from 773 patients with an ovarian tumor and evaluating whether this correlated with clinicopathological parameters and prognosis.

\section{Materials and methods}

Study population. The MALOVA study ('MALignant OVArian cancer study') is a multidisciplinary Danish study covering epidemiology (lifestyle factors), biochemistry and molecular biology with the purpose of identifying risk factors and prognostic factors for OC. The design of the MALOVA study is described in detail elsewhere (14-16). Briefly, preoperative blood samples as well as tumor tissue samples were obtained from most of the patients with a primary epithelial ovarian tumor. FIGO stages were obtained from clinical records and were reviewed by two gynaecologists, both specialized in OC. In the clinical records patients were either classified as radically operated with no macroscopic residual tumor or non-radically operated with macroscopic residual tumor after surgery. On the retrospectively collected paraffin-embedded tissues, histological grading of the tumors was performed individually by two persons. A total of 681 OC and 235 borderline ovarian tumors (LMP) were included in the MALOVA study.
The study was approved by the scientific ethics committee in the study area (KF01-384/95).

Follow-up. In Denmark, all inhabitants have a unique personal (10-digit) identification number (CPR-number), which is used universally in the Danish society. This identification number, which gives information on date of birth and sex, is registered in the computerized Danish National Central Population Register. All OC cases in this study were traced in the register, and date of death, emigration up to October 20, 2004, whichever came first, were registered. In addition, all women who died during follow-up were linked to a Danish hospital reference system, and information on hospital admissions to departments of oncology and/or gynaecology was obtained. The relevant hospital files were collected and scrutinized, and information on treatment (surgery and chemotherapy) and cause of death was established. In cases where the cause of death was uncertain according to the patient file, the women were linked to the Danish Causes of Death Register. At the end of follow-up, a total of 426 OC patients had died from OC (median follow-up time, 23 months; range, 1-111), 232 OC patients were still alive (median follow-up time, 91 months; range, 66-120), 21 OC patients had died from other causes, and no information was available on $2 \mathrm{OC}$ patients.

Tissue array (TA). Paraffin-embedded tissue from the primary surgery was used for TA analyses. Forty-five OC and 24 LMP were excluded because of poor tissue quality or lack of tumor tissue in the collected blocks, and 35 cases (27 OC and 8 LMP) were excluded due to unreadable ER or PR expression results, lack of tumor cells in the selected cylinders, loss of tissue during the staining procedure or folding/tearing of sections by the microtone. Finally, 39 non-epithelial tumors were excluded because epithelial and non-epithelial ovarian tumors differ in embryologic and pathologic characteristics. Together, these exclusions left 773 cases (582 OC, 191 LMP) suitable for combined ER and PR tissue expression and survival analyses.

\section{Immunohistochemical analysis}

Tissue preparation and immunostaining. Sections $(2-\mu \mathrm{m})$ (Section Tranfer System, STS, Ergostar HM200, MICROM International GmbH, Walldorf, Germany) from the TA blocks were transferred to glass slides (Dako ChemMate capillary gap microscope slides, $57 \mathrm{~mm}$, Dako A/S, Glostrup, Denmark). Slides were stored at $4^{\circ} \mathrm{C}$ for a maximum of 8 days until staining for ER or PR.

Prior to staining, the sections were deparaffinized in xylene and rehydrated in graded dilutions of alcohol. In order to demask antigens within the formalin fixed tissue, sections were pretreated in TEG buffer $\mathrm{pH} 9.0$ (Tris $10 \mathrm{mmol} / \mathrm{l}$ and EGTA $0.5 \mathrm{mmol} / \mathrm{l}$ ), followed by heating in a microwave oven at $98^{\circ} \mathrm{C}$ for $15 \mathrm{~min}$. Finally, the sections were cooled by leaving them in the buffer for an additional $15 \mathrm{~min}$.

The staining procedure for ER and PR overexpression was performed on a Dako Techmate ${ }^{\mathrm{TM}} 500^{\text {Plus }}$ instrument using either the monoclonal ER antibody ER1D5 (Immunotech, Marseille, France), at a dilution of 1:200 for $1 \mathrm{~h}$ at room temperature or the monoclonal PR antibody 1A6 (Novocastra 

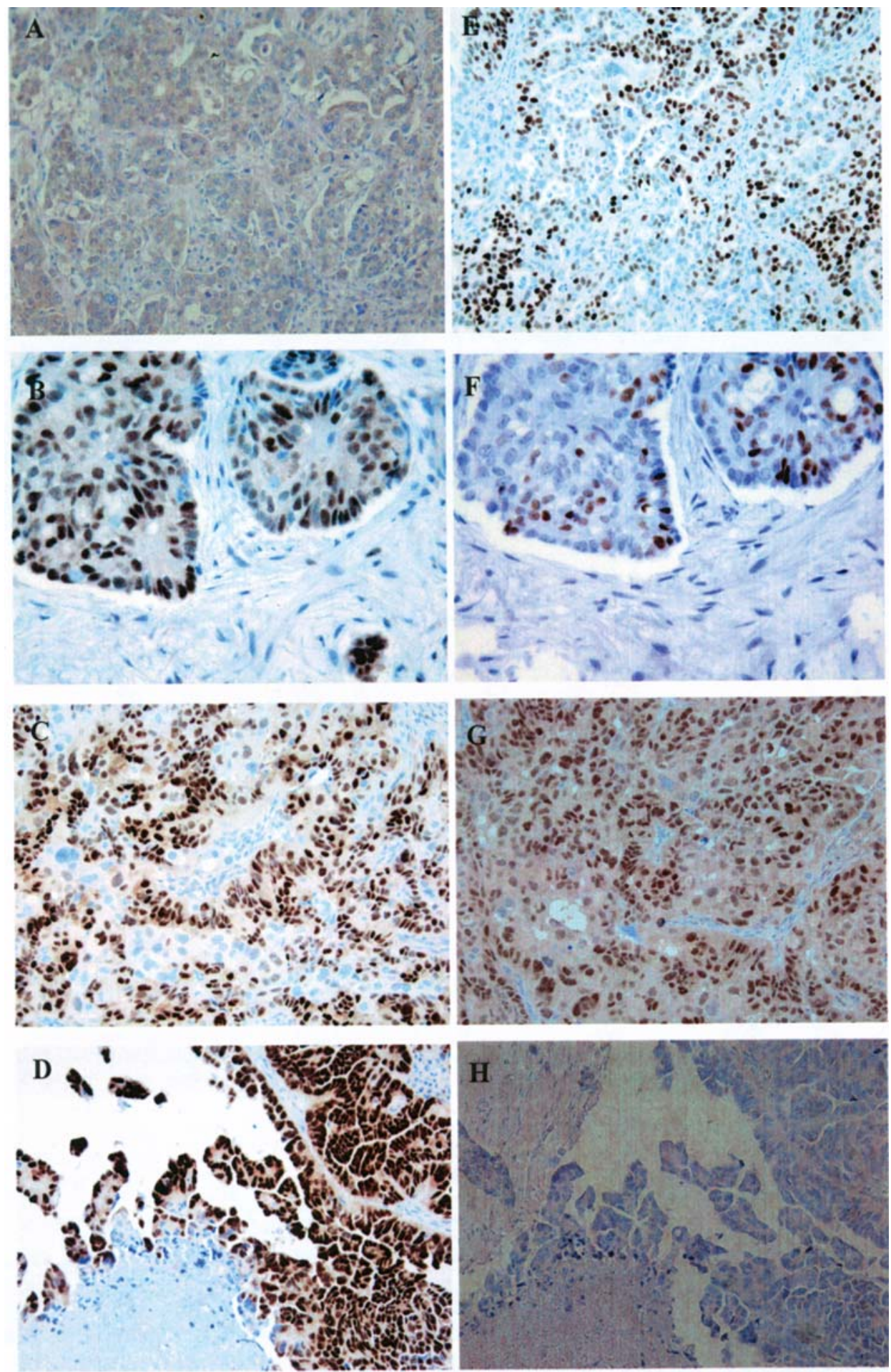

Figure 1. Examples of ER- and PR-stained serous adenocarcinoma tissues from ovarian cancer stage III patients (objective x 40 ). Tissue showing 0\% (A), 40\% (B), $80 \%$ (C) and 100\% (D) ER expression in the tumor cells and the corresponding tissue showing 30\% (E), 20\% (F), 60\% (G) and 0\% (H) PR expression in the tumor cells.

Laboratories, Newcastle, UK), at a dilution of 1:800 for $1 \mathrm{~h}$ at room temperature. Other conditions were set according to the manufacturer's instructions.

ER and PR scoring of tissue expression. Two independent observers, experienced in evaluating immunohistochemically stained tissues, assessed the pattern of staining of ER and PR for each sample. Standardization of scoring was achieved by comparison of the scores between observers, and any discrepancies were resolved by consensus. Scoring for both
ER and PR expression was based on the proportion of cells in a given tumor specimen exhibiting distinct nuclear immunopositivity as well as intensity of staining (percentage scale 0 , $5,10,20,30,40,50,60,70,80,90$ and 100). Secondly, the ER and PR scoring results were transformed into a fourtiered scale (level 1, <10\%; level 2, 10-40\%; level 3, 50$70 \%$; and level $4,>80 \%$ ), and another scale was based on 2 levels of expression results; negative $(<10 \%)$ and positive $(\geq 10 \%)$. 
Representative photomicrographs of tumor tissue showing positive and negative staining for the specific antigen are presented in Fig. 1.

Statistical analyses. Statistical comparisons between groups were carried out using the Chi-square test for categorical data or the rank sum tests for continuous variables. Association between ER tissue expression levels and PR tissue expression levels (percentage) was assessed using the Spearman correlation.

Survival curves were estimated by the Kaplan-Meier method. The impact of ER expression on OC-specific mortality was estimated on the basis of the Cox proportional hazard model (17), applying the four-tiered scale using time since primary surgery as the time axis. Kaplan-Meier survival curves evaluating PR were performed in a similar manner using the same scale. Combinations of the ER and PR expression levels using $10 \%$ as cut-off (ER-PR ${ }^{+}$; ER ${ }^{+} \mathrm{PR}^{-}$; $\mathrm{ER}^{+} \mathrm{PR}^{+}$; ER-PR-) were examined using Kaplan-Meier survival curves.

The assumption of proportionality for the Cox model was assessed using Schoenfeld and martingale residuals as well as graphical methods using SAS, version 9.1. The assumption of proportional hazards between patients not receiving chemotherapy, those treated with platin-based therapy and those treated with other anti-cancer drugs was not fulfilled. Therefore, a stratified proportional hazards model was used for multivariate analysis with strata defined by the therapy group. The regression analyses were adjusted for FIGO stage (I, II, III and IV), residual tumor after primary surgery, histological type of tumor (serous, mucinous, endometroid and other histological types), age at diagnosis (linear) and histological grade. Confidence intervals $(95 \% \mathrm{CI})$ for the corresponding parameters in the multivariate $\mathrm{COX}$ regression model are presented. Patients deceased due to non-related OC diseases were censored in the survival analyses at the date of dead. These analyses were performed using the SPSS 11.5 statistical software.

Inter-observer agreement was assessed by Kappa statistics using SAS, version 9.1. p values $<5 \%$ were considered significant.

\section{Results}

\section{Distribution of ER and PR expression}

Low malignant potential (LMP) ovarian tumors.

ER. Out of 191 LMP ovarian tumors, 161 cases (84\%) were either ER expression negative or with $5 \%$ ER expression (level 1), 25 (13\%) were expression positive with 10-40\% ER expression (level 2), 1 case (1\%) was expression positive with $50 \%$ ER expression (level 3) and the remaining 4 cases ( $2 \%$ ) were positive with $\geq 80 \%$ ER expression (level 4 ) (Table IA).

A tendency towards a difference in the proportion of tumors with $10 \%$ or more ER expression was found among the different FIGO stages $(\mathrm{p}=0.06)$. In addition, a $10 \%$ or higher ER expression was found more frequently in serous than in mucinous LMP tumors $(\mathrm{p} \leq 0.00001)$ (Table IA).

$P R$. Out of 191 LMP tumors, 153 cases $(80 \%)$ were PR expression negative with $<10 \%$ PR expression (level 1), 30
(16\%) were expression positive with $10-40 \%$ PR expression (level 2), 3 cases (2\%) were positive with $50 \%$ or $60 \%$ PR expression (level 3 ) and the remaining 5 cases (2\%) were expression positive with $\geq 80 \%$ PR expression (level 4 ) (Table IB).

A tendency towards a difference in the proportion of tumors with $10 \%$ or more PR expression was found among the different FIGO stages $(\mathrm{p}=0.11)$, although it did not reach statistical significance. A $10 \%$ or higher PR expression was found more frequently in serous than in mucinous LMP tumors $(\mathrm{p} \leq 0.00001)$ (Table IB).

\section{Ovarian cancers.}

ER. Of all 582 OC cases, 375 (64\%) were either ER expression negative or with 5\% ER overexpression (level 1), $142(25 \%)$ were ER positive with 10-40\% (level 2), 45 (8\%) were expression positive with either $50-70 \%$ ER overexpression (level 3) and the remaining 20 (3\%) were ER expression positive with $\geq 80 \%$ (level 4) (Table IA).

The frequency of ER-positive tumors was significantly associated with increasing FIGO stage $(\mathrm{p}=0.0003)$. A higher percentage of ER-positive tumors was also observed among serous $(43 \%)$ than among mucinous $(4 \%)$ types. A significantly different distribution of ER-positive and -negative tumors was found in serous adenocarcinomas, mucinous adenocarcinomas, endometroid adenocarcinomas and other histological subtypes (clear-cell neoplasms, adenocarcinomas NOS and undifferentiated carcinoma) ( $\mathrm{p} \leq 0.00001)$ (Table IA). No significant association was found between grade of tumor (grade 1, 2 or 3 ) and ER expression $(\mathrm{p}=0.40)$. In contrast, a significant association was found between residual tumor after surgery and ER expression ( $\mathrm{p}=0.0005)$ (Table IA).

$P R$. In 582 OC cases, 467 cases (80\%) were PR expression negative or with $5 \%$ PR overexpression (level 1), 82 (14\%) were expression positive with $10-40 \%$ PR overexpression (level 2), 17 (3\%) were expression positive with either 50, 60 or $70 \%$ PR overexpression (level 3) and the remaining $16(3 \%)$ were expression positive with $\geq 80 \%$ (level 4) (Table IB).

No significant association between the frequency of PRpositive carcinomas and increasing FIGO stage was found $(\mathrm{p}=0.12)$. A highly significantly different distribution of PR expression-positive and -negative tumors was found in serous adenocarcinoma, mucinous adenocarcinoma, endometroid adenocarcinoma and other histological subtypes (clear-cell neoplasms, adenocarcinomas NOS, undifferentiated carcinoma) ( $\mathrm{p} \leq 0.00001)$ (Table IB). Higher levels of PR expression were significantly correlated with increasing histological grade $(\mathrm{p}=0.0006)$. A significant association was found between residual tumor after surgery and PR expression $(\mathrm{p}=0.02)$ (Table IB).

$E R$ and PR correlations. The correlation between ER (percentage score) and PR (percentage score) was weak (Spearman $\mathrm{r}=0.23 ; \mathrm{p}<0.0001$ ).

ER expression and PR expression in $O C$ tissue and prognosis. A total of 356 out of the 582 OC patients died of OC during follow-up (26 stage I, 30 stage II, 250 stage III and 50 stage IV).

Univariate Kaplan-Meier survival analysis performed on all OC cases (582 patients), using the four-tiered scale for ER 
Table I. Clinical characteristics and ER/PR expression in tumor tissue from patients diagnosed with borderline ovarian tumor and ovarian cancer.

A, ER expression.

\begin{tabular}{|c|c|c|c|c|c|c|}
\hline \multirow[t]{2}{*}{ Characteristic } & \multicolumn{3}{|c|}{ Ovarian cancer $(n=582)$} & \multicolumn{3}{|c|}{ Borderline ovarian tumors $(n=191)$} \\
\hline & $\mathrm{n}$ & Level $1(\%)^{\mathrm{a}}$ & Level 2, 3 and $4^{\mathrm{a}}$ & $\mathrm{n}$ & Level $1(\%)^{\mathrm{a}}$ & Level 2, 3 and $4^{\mathrm{a}}$ \\
\hline \multicolumn{7}{|l|}{ FIGO stage } \\
\hline I & 165 & $122(74)$ & $20,13,10$ & 166 & $143(86)$ & $19,1,3$ \\
\hline II & 61 & $43(70)$ & $12,4,2$ & 5 & $5(100)$ & - \\
\hline III & 303 & $182(60)$ & $93,21,7$ & 20 & $13(65)$ & $6,0,1$ \\
\hline IV & 53 & $28(53)$ & $17,7,1$ & 0 & - & - \\
\hline \multicolumn{7}{|l|}{ Tumor cell type } \\
\hline Undifferentiated carcinoma & 1 & $7(64)$ & $2,1,1$ & - & & \\
\hline Adenocarcinoma, NOS & 28 & $15(54)$ & $11,1,1$ & - & & \\
\hline Serous adenocarcinoma & 359 & $205(57)$ & $110,34,10$ & - & & \\
\hline Mucinous adenocarcinoma & 53 & $51(96)$ & $1,0,1$ & - & & \\
\hline Endometroid adenocarcinoma & 84 & $51(61)$ & $17,9,7$ & - & & \\
\hline Clear-cell carcinoma & 4 & $46(98)$ & $1,0,0$ & - & & \\
\hline Cystadenoma NOS, LMP & - & & & 4 & $4(100)$ & - \\
\hline Serous cystadenoma, LMP & - & & & 98 & $71(72)$ & $22,1,4$ \\
\hline Mucinous cystadenoma, LMP & - & & & 87 & $84(97)$ & $3,0,0$ \\
\hline Endometroid cystadenoma, LMP & - & & & 2 & $2(100)$ & - \\
\hline \multicolumn{7}{|l|}{ Residual tumor after surgery } \\
\hline Yes & 229 & $167(73)$ & $33,19,10$ & - & & \\
\hline No & 352 & $207(59)$ & $109,26,10$ & - & & \\
\hline No information & 1 & 1 & - & - & & \\
\hline \multicolumn{7}{|l|}{ Histological grade } \\
\hline Grade 1 & 145 & $86(59)$ & $38,16,5$ & & & \\
\hline Grade 2 & 214 & $159(74)$ & $32,13,10$ & & & \\
\hline Grade 3 & 222 & $130(59)$ & $71,16,5$ & & & \\
\hline No information & 1 & $-(0)$ & $1,-,-$ & & & \\
\hline
\end{tabular}

${ }^{a}$ Level 1: 0 and 5\% ER overexpression; level 2: 10, 20, 30 and 40\% ER overexpression; level 3: 50, 60 and 70\% ER overexpression; and level 4: 80, 90 and 100\% ER overexpression.

B, PR expression.

\begin{tabular}{|c|c|c|c|c|c|c|}
\hline \multirow[t]{2}{*}{ Characteristic } & \multicolumn{3}{|c|}{ Ovarian cancer $(n=582)$} & \multicolumn{3}{|c|}{ Borderline ovarian tumors $(n=191)$} \\
\hline & $\mathrm{n}$ & Level $1(\%)^{\mathrm{a}}$ & Level 2, 3 and $4^{\mathrm{a}}$ & $\mathrm{n}$ & Level $1(\%)^{\mathrm{a}}$ & Level 2, 3 and $4^{\mathrm{a}}$ \\
\hline \multicolumn{7}{|l|}{ FIGO stage } \\
\hline I & 165 & $126(76)$ & $24,5,10$ & 166 & $138(83)$ & $22,2,4$ \\
\hline II & 61 & $49(80)$ & $7,3,2$ & 5 & $3(60)$ & $2,-,-$ \\
\hline III & 303 & $247(82)$ & $44,8,4$ & 20 & $12(60)$ & $6,1,1$ \\
\hline IV & 53 & $45(53)$ & $7,1,0$ & - & & \\
\hline \multicolumn{7}{|l|}{ Tumor cell type } \\
\hline Undifferentiated carcinoma & 11 & $10(91)$ & $1,0,0$ & - & & \\
\hline Adenocarcinoma, NOS & 28 & $21(75)$ & $6,0,1$ & - & & \\
\hline Serous adenocarcinoma & 359 & $291(81)$ & $59,7,2$ & - & & \\
\hline Mucinous adenocarcinoma & 53 & $50(94)$ & $0,1,2$ & - & & \\
\hline Endometroid adenocarcinoma & 84 & $50(59)$ & $15,9,10$ & - & & \\
\hline Clear-cell carcinoma & 47 & $45(96)$ & $1,0,1$ & - & & \\
\hline Cystadenoma NOS, LMP & - & & & 4 & $4(100)$ & - \\
\hline Serous cystadenoma, LMP & - & & & 98 & $62(63)$ & $29,3,4$ \\
\hline
\end{tabular}


B, Continued.

\begin{tabular}{|c|c|c|c|c|c|c|}
\hline \multirow[t]{2}{*}{ Characteristic } & \multicolumn{3}{|c|}{ Ovarian cancer $(\mathrm{n}=582)$} & \multicolumn{3}{|c|}{ Borderline ovarian tumors $(n=191)$} \\
\hline & $\mathrm{n}$ & Level $1(\%)^{\mathrm{a}}$ & Level 2, 3 and $4^{a}$ & $\mathrm{n}$ & Level $1(\%)^{\mathrm{a}}$ & Level 2, 3 and $4^{\mathrm{a}}$ \\
\hline Mucinous cystadenoma, LMP & - & & & 87 & $85(98)$ & $1,0,1$ \\
\hline Endometroid cystadenoma, LMP & - & & & 2 & $2(100)$ & - \\
\hline \multicolumn{7}{|l|}{ Residual tumor after surgery } \\
\hline Yes & 229 & $173(76)$ & $35,8,13$ & & & \\
\hline No & 352 & $293(83)$ & $47,9,3$ & & & \\
\hline No information & 1 & 1 & - & - & & \\
\hline \multicolumn{7}{|l|}{ Histological grade } \\
\hline Grade 1 & 145 & $103(71)$ & $30,8,4$ & & & \\
\hline Grade 2 & 214 & $173(81)$ & $27,6,8$ & & & \\
\hline Grade 3 & 222 & $191(87)$ & $25,3,3$ & & & \\
\hline No information & 1 & 0 &,,-- 1 & & & \\
\hline
\end{tabular}

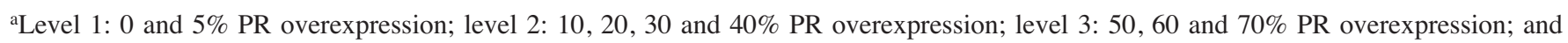
level 4: 80,90 and $100 \%$ PR overexpression.

\section{Survival fraction}

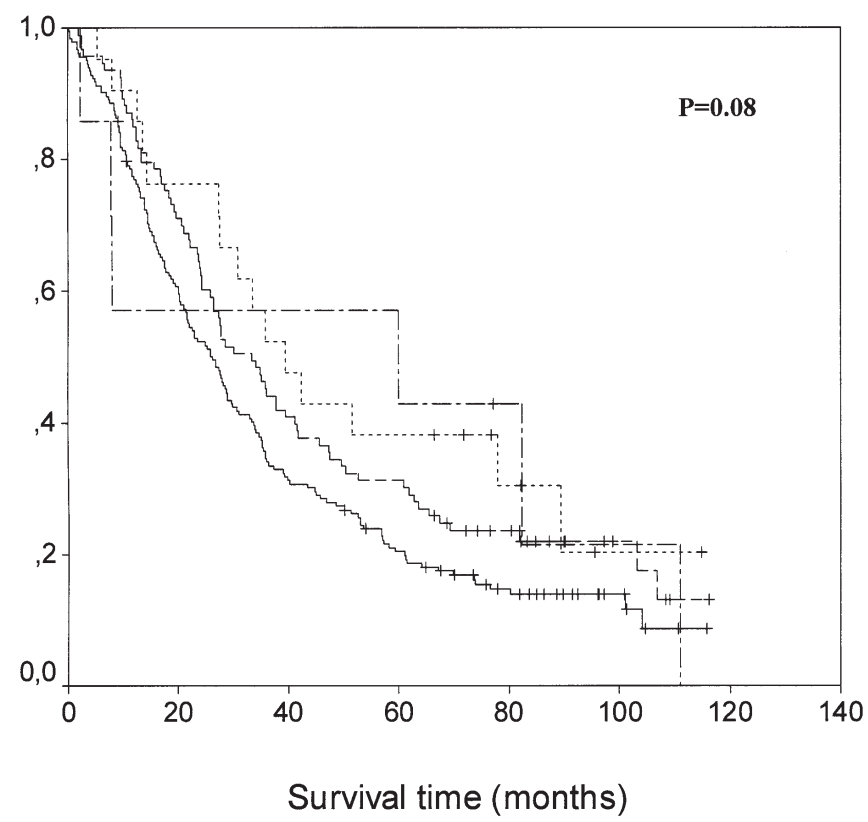

Figure 2. Kaplan-Meier ovarian cancer-specific survival curves. The graph indicates 303 patients who had ovarian carcinoma stage III with $<10 \%$ ER expression $(\mathrm{n}=182$, _ $)$, with $10 \%$ to $<50 \%$ ER expression $(\mathrm{n}=93,----)$, with $50 \%$ to $<80 \%$ ER expression $(n=21, \cdots \cdots)$ and with $80 \%$ or more ER expression in the tumor tissue $\left(n=7,--_{--}\right)$.

expression, showed a significant difference in the survival among OC patients with the four different levels of ER expression $(\mathrm{p}=0.023)$ (data not shown). A shorter survival was found in patients with $10 \%$ to less than $50 \%$ tissue ER expression. The shorter survival of patients with ER tissue expression of 10 to $50 \%$ (level 2) compared to the survival of patients with $<10 \%$ ER expression may be explained by

\section{Survival fraction}

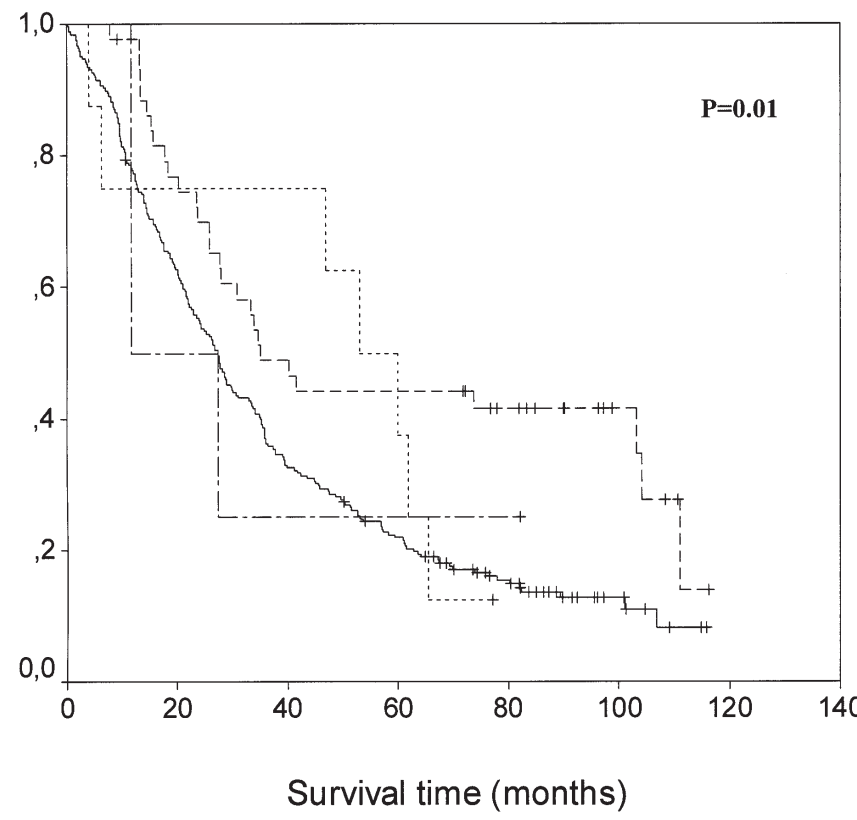

Figure 3. Kaplan-Meier ovarian cancer-specific survival curves. The graph indicates 303 patients who had ovarian carcinoma stage III with $<10 \%$ PR expression $(\mathrm{n}=247,-(-)$, with $10 \%$ to $<50 \%$ PR expression $(\mathrm{n}=44,-----)$, with $50 \%$ to $<80 \%$ PR expression $(n=8, \cdots)$ and with $\geq 80 \%$ PR expression in the tumor tissue $(n=4,-\cdot-\cdot)$.

confounding background variables. As very few OC cases, stage I, II and IV, had $>40 \%$ ER-positive cells (Table IA), only univariate Kaplan-Meier survival analysis stratified by FIGO stage III OC cases is shown in Fig. 2. The univariate survival analysis demonstrated a longer disease-specific survival for patients with $10 \%$ or more ER expression-positive cells ( $\mathrm{p}=0.08)$ (Fig. 2). 


\section{Survival fraction}

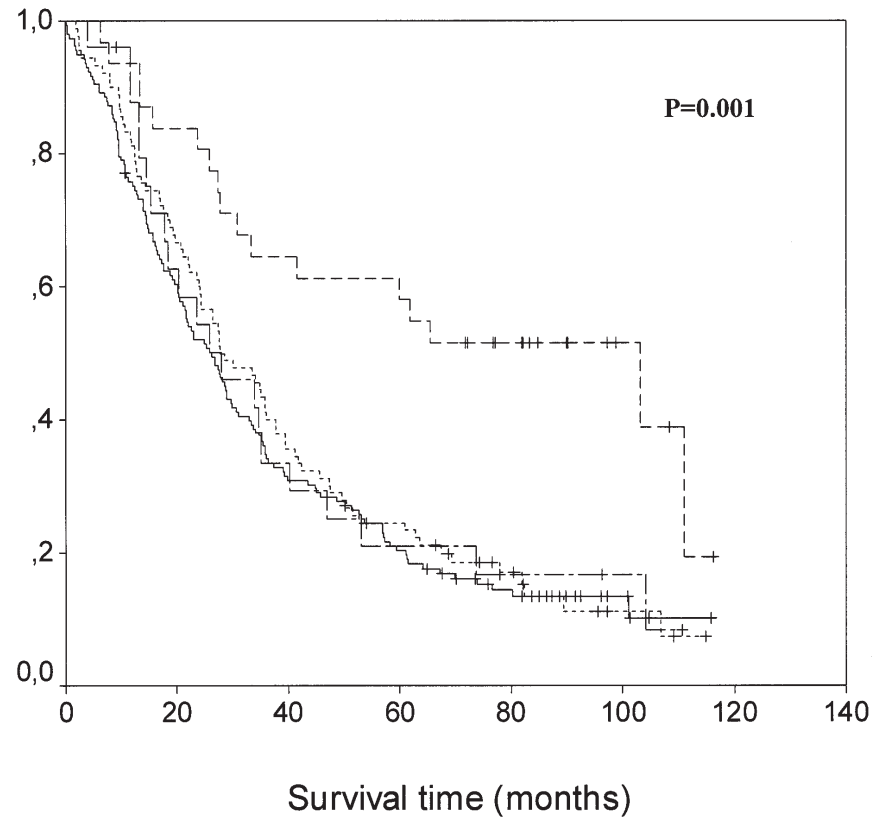

Figure 4. Kaplan-Meier ovarian cancer-specific survival curves. The graph indicates 303 patients who had ovarian carcinoma stage III with $\mathrm{ER}^{+} \mathrm{PR}^{+}$ receptor combination $(n=31,---)$, with $E R \cdot P R^{+}$receptor combination $(n=25$, $\left.-_{--}^{-}\right)$, with ER-PR- receptor combination $(\mathrm{n}=157,-\infty)$ and with $\mathrm{ER}^{+} \mathrm{PR}-$ receptor combination in the tumor tissue $(n=90, \cdots \cdot)$.

In respect to $\mathrm{PR}$ expression, the Kaplan-Meier survival analysis performed on all OC cases (582 patients) using the four-tiered scale for PR expression showed a significant difference in the survival of OC patients with PR expression between the four different levels ( $\mathrm{p}=0.0005)$ (data not shown). Patients with $<10 \%$ PR-positive tumor cells had a shorter survival than patients with higher PR expression. As very few OC cases, stage I, II and IV, had $>40 \%$ PR-positive cells (Table IB), only univariate Kaplan-Meier survival analysis stratified by FIGO stage III OC cases is shown in Fig. 3. The univariate survival analysis demonstrated a longer diseasespecific survival for patients with $10 \%$ or more PR expressionpositive cells ( $\mathrm{p}=0.01$ ) (Fig. 3).

The receptor combinations $\left(\mathrm{ER}-\mathrm{PR}^{+} ; \mathrm{ER}+\mathrm{PR}-\mathrm{ER}^{+} \mathrm{PR}^{+}\right.$; ER-PR-) were also tested in univariate life table analyses. The most common pattern was that of lacking expression for both receptors $(55 \%, 322 / 582)$. An $\mathrm{ER}^{+} \mathrm{PR}^{-}$combination was found in $25 \%(145 / 582)$ and an ER-PR ${ }^{+}$combination was found in $9 \%(53 / 582)$. Coexpression of both receptors $\left(\mathrm{ER}^{+} \mathrm{PR}^{+}\right)$was found in $11 \%$ of the carcinomas (62/582). For stage III OC the expression combinations $\mathrm{ER}^{+} \mathrm{PR}^{+}$versus $\mathrm{ER} \cdot \mathrm{PR}^{+}$were associated with significant differences in disease-specific survival (HR, 0.40; 95\% CI, 0.21-0.76) in contrast to the combinations ER-PR- or ER+PR- versus ER-PR ${ }^{+}$which did not reach statistical significance (ER-PR- versus ER-PR+: HR, 1.10; 95\% CI, 0.69-1.74; ER+PR- versus ER-PR+: HR, 0.99; 95\% CI, 0.61-1.60) (Fig. 4).

In the Cox survival analysis, including 580 stage I to IV OC cases (1 patient without information on residual tumor after surgery and 1 patient with no information of histological grade were excluded) ER and PR tissue expression levels
Table II. Multivariate analysis (stratified by adjuvant therapy) of prognostic factors on all ovarian cancer patients $(n=580)$.

\begin{tabular}{|c|c|c|c|}
\hline Variables & Hazard ratio $^{\mathrm{a}}$ & $95 \% \mathrm{CI}$ & $\mathrm{p}$ value \\
\hline \multicolumn{4}{|l|}{ ER receptor } \\
\hline$<10 \%$ & 1.00 & - & \multirow[t]{2}{*}{0.0490} \\
\hline$\geq 10 \%$ & 0.80 & $0.63-0.99$ & \\
\hline \multicolumn{4}{|l|}{ PR receptor } \\
\hline$<10 \%$ & 1.00 & - & 0.0200 \\
\hline$\geq 10 \%$ & 0.69 & $0.51-0.94$ & - \\
\hline \multicolumn{4}{|l|}{ FIGO stage } \\
\hline II vs. I & 2.88 & $1.45-5.50$ & \multirow[t]{3}{*}{$<0.0001$} \\
\hline III vs. II & 2.49 & $1.67-3.73$ & \\
\hline IV vs. III & 1.87 & $1.35-2.58$ & \\
\hline \multicolumn{4}{|c|}{$\begin{array}{l}\text { Residual tumor after } \\
\text { primary surgery }\end{array}$} \\
\hline No & 1.00 & - & \multirow[t]{2}{*}{$<0.0001$} \\
\hline Yes & 2.80 & $1.89-4.13$ & \\
\hline $\begin{array}{l}\text { Age at diagnosis } \\
\text { (per year) }\end{array}$ & 1.02 & $1.01-1.03$ & 0.0020 \\
\hline \multicolumn{4}{|l|}{ Histology } \\
\hline Serous & 1.00 & - & \multirow{4}{*}{0.1000} \\
\hline Mucinous & 0.81 & $0.48-1.37$ & \\
\hline Endometroid & 1.08 & $0.73-1.60$ & \\
\hline Other types & 1.44 & $1.04-2.00$ & \\
\hline \multicolumn{4}{|c|}{$\begin{array}{l}\text { Histological grade } \\
\text { of tumor }\end{array}$} \\
\hline Grade 1 & 1.00 & - & \multirow[t]{3}{*}{0.3300} \\
\hline Grade 2 & 1.13 & $0.82-1.55$ & \\
\hline Grade 3 & 1.26 & $0.92-1.74$ & \\
\hline
\end{tabular}

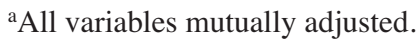

$>10 \%$ were both found to imply an independent significantly favorable course of disease-specific survival (ER: HR, 0.80; 95\% CI, 0.63-0.99; PR: HR, 0.69; 95\% CI, 0.51-0.94), FIGO stage (II vs. I: HR, 2.88; 95\% CI, 1.45-5.50; III vs. II: HR, 2.49; $95 \%$ CI, 1.67-3.73; IV vs. III: HR, 1.87 ; $95 \%$ CI, $1.35-$ $2.58)$, residual tumor after primary surgery (HR, 2.80; $95 \%$ $\mathrm{CI}, 1.89-4.13)$, young age at diagnosis (HR, 1.02; 95\% CI, 1.01-1.03) and other histological types vs. serous adenocarcinoma (mucinous vs. serous: HR, 0.81 ; 95\% CI, 0.48-1.37; endometroid vs. serous: HR, 1.08; 95\% CI, 0.73-1.60; other types vs. serous: HR, 1.44; 95\% CI, 1.04-2.00). Histological grade of tumor $(\mathrm{p}=0.33)$ was found to have no independent prognostic value (Table II).

A test for interaction between ER and PR did not result in a significant interaction $(\mathrm{p}=0.19)$. The results of the multivariate model suggest that the prognostic value of ER and PR are additive. The hazard ratio for patients with high ER and PR expression was 0.48 (95\% CI, 0.31-0.74; $\mathrm{p}=0.001)$ compared to patients with $<10 \%$ for both receptors. This was also significantly lower than the hazard ratio for patients who had only one of the receptors elevated $(\mathrm{p}=0.046)$. 
Control on immunostaining quality and re-evaluation. An inter-observer discrepancy in tissue ER expression at the percentage level occurred in 28 out of 773 cases $(3.6 \%)$. Similarly, a discrepancy in PR expression at the percentage level occurred in 21 out of 773 cases $(2.7 \%)$. None of the discrepancies exceeded more than one level in the four-tiered scale. All immunostaining results not in agreement were mutually discussed and a consensus percentage result was obtained. The Kappa statistic for ER was 0.98 (95\% CI, 0.960.99) and for PR 0.96 (95\% CI, 0.94-0.99).

\section{Discussion}

In this study we used TA analysis to analyze a large series of ovarian tumor tissue samples for ER and PR expression and clinical patient follow-up to evaluate the prognostic value of the two markers. The prognostic value of both ER and PR has been debated for years and is still controversial. One explanation for the difference in results may lie in the heterologous composition of patients enrolled in the studies, the number of samples analysed, the different detection methods, and differences in antibodies, but it may also lie in the different criteria for the scoring of the histological findings. Immunohistochemistry is today considered a valuable evaluation method, because it allows an exact assignment of ER and PR expression to tissue components of interest $(4,5,8,9,18,19)$.

To our knowledge this is the largest study describing the relationships between ER and PR expression in ovarian tumor tissue and also with respect to examine the prognostic values of the receptor status in OC.

The strengths of our well-characterized study group include the large number of tissue samples investigated and no loss to follow-up. The MALOVA samples include both LMP tumors and invasive disease of different histological subtypes, and the large number of cases including both early stage disease and advanced stage disease provided us with the ability to perform a reliable prognostic evaluation.

A simultaneous expression of ER and PR in epithelial cells of the histopathological tumor types other than mucinous adenocarcinoma and clear-cell carcinoma was clearly observed for both LMP tumors and OC, although more distinct for OC. This is in accordance with a previous report, which also found ER and PR poorly expressed by mucinous carcinomas (4). Another large OC study by Lee et al demonstrated that only $\mathrm{PR}$, in contrast to $\mathrm{ER}$, is found to be associated with a high degree of differentiation, early stage and long survival (5). These findings are in contrast to our results, which did not indicate any correlation between PR and stage but instead saw a significant increase in ER expression with higher stage. One reason for this discrepancy may be the comparatively few early stage OCs included in the study by Lee et al (5).

Reported frequencies of ER and PR expression vary from 62 to $77 \%$ for ER and from 26 to $43 \%$ for PR, respectively $(5,9)$. Our findings reflect particularly low frequencies for OC with only 36 and $20 \%$ being positive for ER and PR, respectively. In LMP tumors the expression frequencies were found similar for both receptors (16\% for ER and $20 \%$ for $\mathrm{PR})$. This may indicate that LMP tumors and OC overall do not reflect a continuum of disease progression but rather different disease entities. One study reported that ER and PR are mutually correlated (9), and that PR is inversely correlated with stage and high degree of differentiation (9); findings in accordance with a study by Lee et al (5). In agreement, we found PR to be correlated inversely with histological grade of tumor and lower PR expression to correlate with higher stage of disease.

Few studies have evaluated the prognostic value of ER and PR. Münstedt et al found that ER alone, in contrast to PR alone, had no value (9), but by examining the combined ER/ PR expression, they found that in particular ER-negative/PRpositive $(>10 \%)$ tumors were associated with an increased overall survival for the patients (9). Another study also reported PR expression to be related to improved survival, results that were explained by the mechanisms of the progesterone-mediated inhibition of cell proliferation and induced apoptosis (20). Although one study failed to demonstrate any prognostic value of the ER receptor, it reported that patients with PR expression $>10 \%$ survived longer than patients with PR expression levels $<10 \%$ (5).

We observed similar findings for PR when performing univariate survival analyses, and in our multivariate survival analyses on OC we further found that increasing ER or PR tissue expression served as an independent prognostic factor for longer disease-specific survival. As our evaluations of receptor combinations further suggested $\mathrm{ER}^{+} \mathrm{PR}^{+}$to have an additive impact on survival, both biomarkers are most likely indicators of a favorable prognosis in patients with OC.

Several factors may influence biomarker expression in tumor samples. Differences in tissue sampling and processing may cause variations in expression levels, and heterogeneity of steroid receptor expression within a tumor may question whether the sample is representative of the whole tumor. We used TAs of a large diameter $(2 \mathrm{~mm})$ in order to minimize this problem. The two persons who scored all the array samples did not have $>3 \%$ discrepancy between ER expression scores and had $<4 \%$ discrepancy between PR expression scores. The core size of the tissue samples, the design of the TAs and the opportunity to be able to score up to four different cores from each sample are all important factors to be considered in array production.

In conclusion, our results show that overexpression of ER and $\mathrm{PR}$, alone and in combination, may predict a favorable outcome in patients with OC. As OC tumorigenesis is complex, it is conceivable that more potential prognostic markers will emerge in the coming years, but it is still important to identify reliable prognostic markers, such as ER and PR in tumor tissue, not only as possible future targets of therapy but also as predictive markers for selection of patients for individualized treatments in future $\mathrm{OC}$ trials.

\section{Acknowledgements}

We thank all the nurses and doctors of the gynecological and pathological departments for their tremendous work. The authors are grateful to Heidi Marie Paulsen, Vibeke Reese and Connie Kjelkvist for their technical assistance. Kirsten Frederiksen is thanked for the fruitful statistical discussions. This study was supported by grants from Mermaid1, Aase and Ejnar Danielsens foundation, The Danish Cancer Society 
and National Cancer Institute, Bethesda, MD, USA (RO1 CA 61107).

\section{References}

1. Kjaerbye-Thygesen A, Huusom LD, Frederiksen K and Kjaer SK: Trends in the incidence and mortality of ovarian cancer in Denmark 1978-2002 - Comparison with other Nordic countries. Acta Obstet Gynecol Scand 84: 1006-1012, 2005.

2. Lu KH, Patterson AP, Wang L, et al: Selection of potential markers for epithelial ovarian cancer with gene expression arrays and recursive descent partition analysis. Clin Cancer Res 10: 3291-3300, 2004.

3. Rapkiewicz AV, Espina V, Petricoin EF and Liotta LA: Biomarkers of ovarian tumours. Eur J Cancer 40: 2604-2612, 2004.

4. Lindgren PR, Cajander S, Bäckström T, Gustafsson JA, Mäkelä S and Olofsson JI: Estrogen and progesterone receptors in ovarian epithelial tumors. Mol Cell Endocrinol 221: 97-104, 2004.

5. Lee P, Rosen DG, Zhu C, Silva EG and Liu J: Expression of progesterone receptor is a favorable prognostic marker in ovarian cancer. Gynecol Oncol 96: 671-677, 2005.

6. Syed V, Zhang X, Lau KM, Cheng R, Mukherjee K and Ho SM: Profiling estrogen-regulated gene expression changes in normal and malignant human ovarian surface epithelial cells. Oncogene 24: 8128-8143, 2005

7. Bardin A, Hoffmann P, Boulle N, Katsaros D, Vignon F, Pujol P and Lazennec G: Involvement of estrogen receptor beta in ovarian carcinogenesis. Cancer Res 64: 5861-5869, 2004.

8. Lazennec G: Estrogen receptor beta, a possible tumor suppressor involved in ovarian carcinogenesis. Cancer Lett 231: 151-157, 2006.

9. Münstedt K, Steen J, Knauf AG, Buch T, von Georgi R and Franke FE: Steroid hormone receptors and long term survival in invasive ovarian cancer. Cancer 89: 1783-1791, 2000.

10. Kleine W, Maier T, Geyer H and Pfleiderer A: Estrogen and progesterone receptors in endometrial cancer and their prognostic relevance. Gynecol Oncol 38: 59-65, 1990
11. Iwai K, Fukuda K, Hachisuga T, Mori M, Uchiyama M, Iwasaka T and Sugimori H: Prognostic significance of progesterone receptor immunohistochemistry for lymph node metastases in endometrial carcinoma. Gynecol Oncol 72: 351-359, 1999.

12. Kumar B, De Silva M, Venter DJ and Armes JE: Tissue microarrays: a practical guide. Pathology 36: 295-300, 2004.

13. Nocito A, Kononen J, Kallioniemi OP and Sauter G: Tissue microarrays (TMAs) for high-throughput molecular pathology research. Int J Cancer 94: 1-5, 2001

14. Høgdall EV, Christensen L, Kjaer SK, et al: Distribution of HER-2 overexpression in ovarian carcinoma tissue and its prognostic value in patients with ovarian carcinoma: from the Danish MALOVA Ovarian Cancer Study. Cancer 98: 66-73, 2003.

15. Høgdall EV, Ryan A, Kjaer SK, et al: Loss of heterozygosity on the $\mathrm{X}$ chromosome is an independent prognostic factor in ovarian carcinoma: from the Danish 'MALOVA' Ovarian Carcinoma Study. Cancer 9: 2387-2395, 2003.

16. Kjaerbye-Thygesen A, Frederiksen K, Høgdall EV, et al: Smoking and overweight: negative prognostic factors in stage III epithelial ovarian cancer. Cancer Epidemiol Biomarkers Prev 15: 798-803, 2006.

17. Cox DR: Regression models and life tables. J Roy Stat Soc B 34: 187-220, 1972.

18. Remmele W and Stegner HE: Recommendation for uniform definition of an immunoreactive score (IRS) for immunohistochemical estrogen receptor detection (ER-ICA) in breast cancer tissue. Pathologe 8: 138-140, 1987.

19. van Doorn HC, Burger CW, van der Valk $\mathrm{P}$ and Bonfrèr $\mathrm{HM}$ Oestrogen, progesterone, and androgen receptors in ovarian neoplasia: correlation between immunohistochemical and biochemical receptor analyses. J Clin Pathol 53: 201-205, 2000.

20. Lindgren P, Bäckström T, Mählck CG, Ridderheim M and Cajander S: Steroid receptors and hormones in relation to cell proliferation and apoptosis in poorly differentiated epithelial ovarian tumors. Int J Oncol 19: 31-38, 2001. 